\title{
Why and How Do Black Holes Radiate?
}

\author{
Risto Raitio \\ 02230Espoo, Finland \\ Email: risto.raitio@gmail.com
}

Received 27 November 2015; accepted 14 December 2015; published 18 December 2015

Copyright (C) 2015 by author and OALib.

This work is licensed under the Creative Commons Attribution International License (CC BY). http://creativecommons.org/licenses/by/4.0/

\section{(c) (i) Open Access}

\begin{abstract}
The phenomenological model proposed in this note indicates that the black hole radiation consists of two components: the standard thermal Hawking radiation and an additional non-thermal baryonic/leptonic component due to quantum number neutralization by the no-hair theorem. The particle radiation grows relatively stronger than the Hawking radiation with increasing black hole mass, and it can be tested in principle.
\end{abstract}

\section{Keywords}

Quantum Black Hole, Hawking Radiation, Standard Model, Grand Unified Theory

Subject Areas: Particle Physics

\section{Introduction and Summary}

The purpose of this note is to propose a quantum mechanical model for the total radiation of black holes (BH). The black hole radiation is shown to consist of thermal Hawking radiation and an additional non-thermal baryonic/leptonic component assuming quantum number neutralization by the no-hair theorem. I discuss the logical structure of the model rather than give numerical predictions at this stage.

Particles fall into the hole through the horizon. The effective horizon extends above the the Schwarzschild value by a factor of $\frac{3 \sqrt{3}}{2}$ [1]. If the black hole mass is at or above the Planck scale the particle enters the grand unified theory (GUT) energy domain. Most arguments of this note also apply if only the standard model is considered. Inside the hole the fallen particle looses its quantum numbers except for charge, mass and spin due to the no-hair theorem. Lepton and baryon number neutralization takes place via radiation as follows. For example, a fallen electron inside the hole can observe a virtual electron-positron pair being formed just outside the horizon. The positron is taken inside the hole neutralizing the electron into energy like photons or ultimately gravitons. Thus a fallen electron causes another electron being emitted as if from the hole. The same happens to a fallen proton unless it first decays into an electron and a pion.

A compatible decay model of black holes of Planck mass has been introduced in [2] [3]. In the last phase of 
radiation before disappearance of the hole the basic element of the model is the gravon [2], a critical quantum state which connects the black hole to a quantum field theory. Instead of vanishing totally after radiating off all its energy, a micro black hole, with mass of about the Planck mass value, triggers the operators of the standard model (SM) or a grand unified quantum field theory like SO (10). Thereafter the black hole energy decays into $\mathrm{SO}(10)$ particles and finally into standard model particles. Primordial black holes (PBH) with mass about $10^{15} \mathrm{~g}$ will be evaporating today and their abundance is constrained by the flux of gamma-rays, for a comprehensive treatment see [4].

A model of corpuscular structure of black holes is needed for the basis of the model. Such a model has been presented in [5] though we do not rely on its details. The model is a Bose-Einstein condensate (BEC) of gravitons interacting in a deep well whose depth is proportional to the number $N$ of gravitons present. For large $\mathrm{N}$ the gravitons can superimpose forming a ball of characteristic radius

$$
\lambda \sim \frac{\hbar}{m}=L_{\text {Planck }} \frac{M_{\text {Planck }}}{m}
$$

where $m$ is the effective graviton mass. When each photon has kinetic energy just below the well depth the ball of gravitons becomes a self-confined black hole. Graviton excitations can lead to quantum depletion of the condensate which is interpreted as Hawking radiation in [5].

The SO (10) GUT gets support from a different direction, and time, in the universe. In [6] the authors study Starobinsky inflation in a renormalizable grand unified theory based on the SO (10) gauge group with no scale supergravity theory (SUGRA).

In this note I discuss the physical motivation and description of the model. Section 2 discusses the radiation mechanisms of black holes. Section 3 mentions briefly thoughts of black holes being condensates of gravitons and other massless fields. In Section 4 I describe the gravon qualitatively. Section 5 summarizes briefly an SO (10) GUT inflation model with a rich multiplet structure. I finish in Section 6 with conclusions. Sections 3 and 5 are very brief surveys of work done by other authors. They are included for consistency of the framework and as directions of future work.

\section{Black Hole Total Radiation}

Particles falling into a black hole through the horizon is a "no-drama" event. The hole is supposed to have no hair, though. When a proton, or other baryon, falls into a black hole it should neutralize (barber) its baryon number into energy like gravitons.

Within the standard model this takes place by absorbing an antiproton of a virtual pair from near the horizon, which gives minimal growth of mass of the hole. In a GUT, the proton inside the horizon of the hole may first decay into lighter particles, $p \rightarrow e^{+} \pi$, and subsequently the positron lepton number is neutralized by an electron from a virtual pair leading to faster growth of the hole mass. The amplitude for the neutralization is roughly proportional to $A\left(e^{-}+e^{+} \rightarrow\right.$ graviton + graviton $)$ and similarly for baryons. The intensity of the outgoing radiation depends clearly on the flux of particles falling into the black hole.

When a hole is bombarded by baryons or leptons the radiation of the hole is predicted to have a thermal Hawking and baryonic/leptonic component consisting dominantly of the lightest leptons. While a BH gets cooler with increasing mass the relative amount of the stimulated baryonic/leptonic component is expected to increase with the mass of the hole.

\section{Structure of Black Holes}

Using the concepts of the previous section one is lead to consider a corpuscular model of some kind for black holes. One such model has been proposed in [5]. There is a recent review of results within this scheme in [7], obtained by various authors. The basic idea is that a black hole is a Bose-Einstein condensate (BEC) of gravitons. Though we do not need any details of that model in this note we wish to mention one feature of it.

The authors in [8] consider massless scalar gravitons instead of real ones and write a Klein-Gordon equation for them with a real scalar current in Minkowski space

$$
\square \phi(x)=q J(x)
$$

Later an interesting concept called the horizon wave function (HWF) is introduced starting from the energy 
eigenvalue equation

$$
\hat{H}\left|\psi_{E}\right\rangle=E\left|\psi_{E}\right\rangle
$$

HWF is supposed to apply to quantum mechanical states $\psi_{\mathrm{S}}$ of systems localized in space and at rest in the chosen reference frame. Any state $\psi_{\mathrm{S}}$ can be presented using these energy eigenstates

$$
\left|\psi_{\mathrm{S}}\right\rangle=\sum_{E} c(E)\left|\psi_{E}\right\rangle
$$

Inverting the expression of the Schwarzschild radius,

$$
r_{\mathrm{H}}=2 G_{\mathrm{N}} E=2 \frac{L_{\text {Planck }}}{M_{\text {Planck }}} E
$$

one obtains $E$ as a function of $r_{\mathrm{H}}$. The authors in [8] later consider among other things calculating the probability that a particle of width $l$ is a black hole as a function of $l / L_{\text {Planck }}$. Therefore the above expression is used to define the horizon probability amplitude, or wave function in [8], from the the coefficient $c(E)$

$$
\psi_{\mathrm{H}}\left(r_{\mathrm{H}}\right) \propto c(E)=c\left(M_{\text {Planck }} r_{\mathrm{H}} / 2 L_{\text {Planck }}\right)
$$

whose normalization is determined by the inner product

$$
\left\langle\psi_{\mathrm{H}} \mid \phi_{\mathrm{H}}\right\rangle=4 \pi \int_{0}^{\infty} \psi_{\mathrm{H}}^{*}\left(r_{\mathrm{H}}\right) \phi_{\mathrm{H}}\left(r_{\mathrm{H}}\right) r_{\mathrm{H}}^{2} \mathrm{~d} r_{\mathrm{H}}
$$

Starting from the HWF one then goes to define the probability density of finding a gravitational radius $r=r_{\mathrm{H}}$ corresponding to the given quantum state $\psi_{\mathrm{s}}$ as

$$
\mathcal{P}_{\mathrm{H}}\left(r_{\mathrm{H}}\right)=4 \pi r_{\mathrm{H}}^{2}\left|\psi_{\mathrm{H}}\left(r_{\mathrm{H}}\right)\right|^{2}
$$

It is seen that the HWF takes the classical concept of the Schwarzschild radius into the quantum domain. The gravitational radius turns out to be necessarily "fuzzy", since it is related to a quantity, the energy of the particle, that is naturally uncertain.

Provided the BEC model of the black holes is valid the above discussion indicates a possible avenue for further development of the present model.

\section{The Gravon and Decay of Black Holes}

The universe is described by classical general relativity at large distances and by grand unified quantum field theory of particles at microscopic scales. The gravon [2] is a critical connecting state between microscopic black holes and quantum fields. As seen from the quantum field side it is the equivalent of quantum state vector collapse into the classical theory. Seen from the classical side the black hole looses (barbers) its horizon and makes a transition into quantum fields. The horizon requires proper mathematical treatment but the physical picture given here should be clear.

The decay of a micro black hole can be approximated by a heavy Higgs-like scalar (or a heavy fermion) decay. $\mathrm{SO}(10)$ is a group which has a 16 dimensional spinorial representation (16) which can accommodate all one generation quarks and leptons. Therefore at the energy considered, the Planck scale, all particles have zero mass, all interactions have the same strength, all gauge bosons (45) can be produced freely and all quarks can transform into leptons. The Higgs come in the representations (10), (16) and (45).

\section{Inflation}

In [6] the authors study inflation in a renormalizable grand unified theory based on the SO (10) gauge group with no scale SUGRA. The authors show that a renormalizable Wess-Zumino superpotential of SO (10) GUT along with no-scale Kähler potential can produce Starobinsky kind of inflationary potential with specific choice of superpotential parameters.

The Higgs supermultiplets the authors consider are 10, 210, $126(\overline{126})$. Among these, the 210 and $126(\overline{126})$ are responsible for breaking of SO (10) symmetry down to minimal supersymmetric standard model (MSSM). The 210 supermultiplet alone can give different intermediate symmetries [9] depending upon which of its 
MSSM singlet field takes a vacuum expectation value (vev). The $126(\overline{126})$ breaks this intermediate symmetry to MSSM. Successful inflationary potential can be achieved in the case of $S U(3)_{C} \times S U(2)_{L} \times S U(2)_{R} \times U(1)_{B-L}$, $S U(5) \times U(1)$ and flipped $S U(5) \times U(1)$ symmetry. The other possible intermediate symmetries of PatiSalam $\left(S U(4)_{C} \times S U(2)_{L} \times S U(2)_{R}\right)$ or $S U(3)_{C} \times S U(2)_{L} \times U(1)_{R} \times U(1)_{B-L}$ gauge groups do not give phenomenologically correct inflationary potentials.

At the end of inflation reheating can occur via non-perturbative decay of inflaton to bosons of the intermediate scale model. After the end of reheating, when universe cools down, the finite temperature potential can have a minimum which corresponds to MSSM and the universe rolls down to this minimum at temperature $\ll T_{R}$ (reheat temperature).

The Starobinsky model, based on one loop quantum gravity, is known to fit all the CMB data available today. From the point of view of the present model it would be interesting to study whether the calculation of [6] could yield similar results without assuming supersymmetry.

\section{Conclusions}

The present note contains a proposal of a model for black hole particle radiation and decay. The radiation is a consequence of the no-hair theorem requiring baryon and lepton number neutralization. Planck mass black hole decay and black hole radiation are different kinds of processes in this scenario. According to the model, the ratio of thermal Hawking radiation to baryonic/leptonic radiation intensity decreases with increasing black hole mass. These claims are testable in principle.

The SO(10) based GUT model [6] gives a good phenomenological description of the inflationary universe [3]. It seems that one can comprehend early and late periods of life cycle of particles in the (bouncing) universe by using the SO (10) based GUT and the black hole model of this note. ${ }^{1}$

Targets needing future research include the following. The use of grand unified theories near or within black hole horizons and black hole radiation mechanisms should be analyzed in quantitative detail, including stellar size holes. Secondly, corpuscular models of the structure of black holes need to be set on solid basis. Thirdly, the dynamical details of the present simple black hole model and the more involved supersymmetric inflationary model mentioned in Section 5 should be compared and studied.

\section{References}

[1] Giddings, S.B. (2015) Hawking Radiation, the Stefan-Boltzmann Law, and Unitarization. http://arxiv.org/abs/1511.08221

[2] Raitio, R. (2015) The Decay of a Black Hole in a GUT Model. Open Access Library Journal, 2, e2031. http://dx.doi.org/10.4236/oalib.1102031

[3] Raitio, R. (2015) A Standard Model at Planck Scale. http://vixra.org/pdf/1507.0023v4.pdf

[4] Green, A.M. (2014) Primordial Black Holes: Sirens of the Early Universe. http://arxiv.org/abs/1403.1198

[5] Dvali, G. and Gomez, C. (2014) Quantum Compositeness of Gravity: Black Holes, AdS and Inflation. JCAP, No. 01, 023.

[6] Garg, I. and Mohanty, S. (2015) No Scale SUGRA SO (10) Derived Starobinsky Model of Inflation. http://arxiv.org/abs/1504.07725

[7] Casadio, R., Giugno, A., Micu, O. and Orlandi, A. (2015) Thermal BEC Black Holes. http://arxiv.org/abs/1511.01279

[8] Casadio, R., Giugno, A., Micu, O. and Orlandi, A. (2014) Black Holes as Self-Sustained Quantum States and Hawking Radiation. Physical Review D, 90, Article ID: 084040. http://dx.doi.org/10.1103/PhysRevD.90.084040

[9] Aulakh, C.S., Bajc, B., Melfo, A., Senjanovic, G. and Vissani, F. (2004) The Minimal Supersymmetric Grand Unified Theory. Physics Letters B, 588, 196-202. http://dx.doi.org/10.1016/j.physletb.2004.03.031 http://arxiv.org/abs/hep-ph/0306242

\footnotetext{
${ }^{1}$ For a glimpse of theoretical results and problems in quantum gravity see eg. Z. Bern, Ultraviolet Surprises in Gravity, BAPTS, Oct 9, 2015.
} 